\title{
A Complex Cross Link between the Tendons of Flexor Hallucis Longus and Flexor Digitorum Longus
}

\author{
Srinivasa Rao Sirasanagandla ${ }^{1}$ Varna Taranikanti ${ }^{2}$ Raghu Jetti ${ }^{3}$ \\ ${ }^{1}$ Department of Human and Clinical Anatomy, College of Medicine \\ and Health Sciences, Sultan Qaboos University, Muscat, Oman \\ ${ }^{2}$ Department of Foundational Medical Studies, Oakland University \\ William Beaumont School of Medicine, Rochester, MI 48309, USA \\ ${ }^{3}$ Department of Basic Medical Sciences, College of Applied Medical

\begin{abstract}
Address for correspondence Srinivasa Rao Sirasanagandla, MSc, PhD, Department of Human and Clinical Anatomy, College of Medicine and Health Sciences, Sultan Qaboos University, P.O. Box 50, P.C. 123, Muscat, Oman (e-mail: srinivasa@squ.edu.om).
\end{abstract} Sciences, King Khalid University, Abha, Kingdom of Saudi Arabia

J Morphol Sci 2018;35:177-179.

\begin{abstract}
Keywords

- cross link

- flexor digitorum longus

- flexor hallucis longus

- flexor digitorum accessorius

- first lumbrical

- variation

Anatomical variations involving the flexor digitorum longus (FDL) and flexor hallucis longus (FHL) tendons are clinically important because of their significant role in reconstructive surgeries of tibialis posterior tendon insufficiencies and Achilles tendinopathies. Harvesting of grafts from these complex variations of FDL and FHL are surgically challenging. In the present case, we report a complex structural cross link between the FDL and FHL associated with morphological variations of the first lumbrical and flexor digitorum accessorius (FDA) attachments. A connecting slip from FHL was found to be inserted into the FDL tendon of the second toe. The FDA was inserted into the fibular side of the connecting slip of the FHL as well as into the FDL tendons of the third and fourth toes. The first lumbrical took origin from the tibial side of FHL connecting slip and from the tibial side of FDL tendon for second toe.
\end{abstract}

\section{Introduction}

The flexor hallucis longus (FHL) muscle is one among the deep group muscles of the posterior compartment of the leg; it arises from the posterior surface of the lower two thirds of the fibula, lateral to the medial crest. The tendon passes downwards in a groove between the medial and lateral tubercles of the calcaneum and then runs in a groove under the sustentaculum tali; finally, it is inserts itself into the base of the distal phalanx of the great toe. The flexor digitorum longus (FDL) arises from the medial part of the posterior surface of the tibia and is divided into four tendons for the lateral four toes, which are finally inserted into the distal phalanx of the corresponding toes. The flexor digitorum accessorius (FDA) or quadratus plantae muscle arises from the lateral tubercle of the calcaneum and inserted into the tendon of the FDL. ${ }^{1}$

The FHL tendon is frequently used to treat Achilles tendinopathy. It is also the preferred choice for the surgical treatment of chronic posterior tibial tendon deficiency (PTTD). ${ }^{2}$ The anatomic relationship between the tendons of the FHL and
FDL, and the existence of cross links between these tendons are clinically challenging to reconstructive surgeons. A few authors have previously reported and classified the presence of a variety of cross links between the FHL and FDL. ${ }^{3,4}$ However, reporting of this complex cross link of tendons along with associated morphological variations is of high clinical relevance because of the pivotal role they play in reconstructive surgeries. In the present case, we report a complex variation of cross link between the FHL and FDL associated with unusual insertion of the FDA and first lumbrical.

\section{Case Report}

During regular dissection classes for the medical undergraduates, a rare anatomical variation in the left extremity of a male cadaver was seen. A cross link between the FHL and FDL tendons associated with unusual attachments of the FDA and origin of first lumbrical was found. The FHL tendon presented a connecting slip at the level of the junction between the proximal one third and distal two thirds of the sole. The received

March 3, 2017

accepted

September 3, 2018

published online

October 31, 2018
DOI https://doi.org/

10.1055/s-0038-1675225. ISSN 2177-0298.
Copyright $(2018$ by Thieme Revinter

Publicações Ltda, Rio de Janeiro, Brazil
License terms

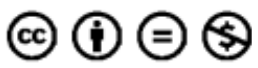


connecting slip coursed laterally under the tendon of the FDL and finally inserted itself into the FDL tendon of the second toe. The FDA was inserted mainly into the fibular side of the connecting slip of the FHL and partly on to the undersurface of the FDL tendons of the third and fourth toes. First lumbrical was bipennate in origin, with fibers arising from the tibial side of the FHL connecting slip and from the tibial side of the FDL tendon to the second toe (-Figs. 1 and $\mathbf{2}$ ).

\section{Discussion}

The accessory slips or connecting slips of the FHL and FDL have been reported in the literature. These interconnections were classified into type I, in which there is a slip from the FHL to FDL; type II-crossed slips between them, that is, from the FHL to FDL, from the FDL to FHL; type III-from the FDL to FHL; and type IV, in which there is no slip between them. ${ }^{5,6}$ Type I is further classified based on the tendons of the toes to which it is connected; there is type Ia, in which the slip is attached to the second toe tendon; type Ib-slip attached to the second and third toe tendons; type Ic-slip attached to the second, third and fourth tendons; and type Id, in which the slip is attached to the tendons of all toes, except for the

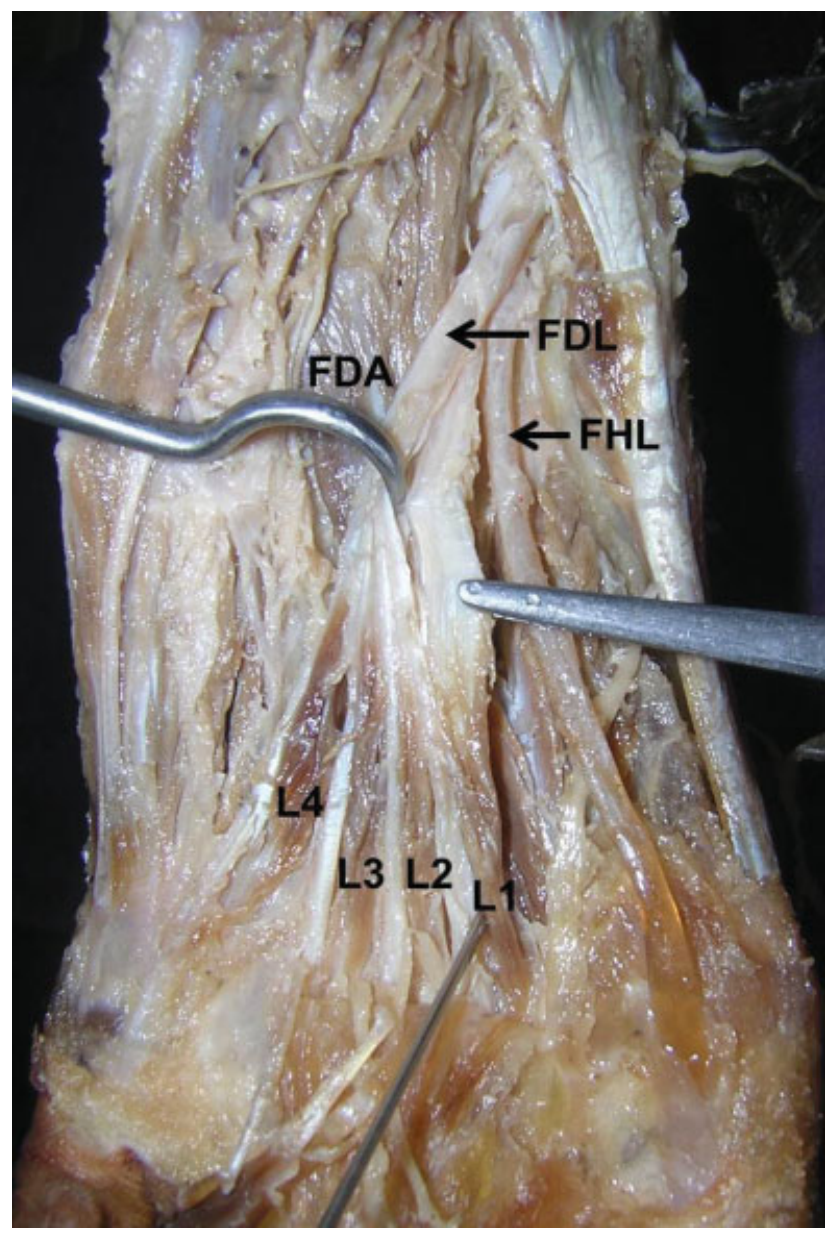

Fig. 1 Dissection of foot showing the intrinsic muscles and extrinsic tendons of the second layer of the sole. Abbreviations: FDL, flexor digitorum longus; FHL, flexor hallucis longus; FDA, flexor digitorum accessorius; L1, first lumbrical; L2, second lumbrical; L3, third lumbrical; L4, fourth lumbrical.

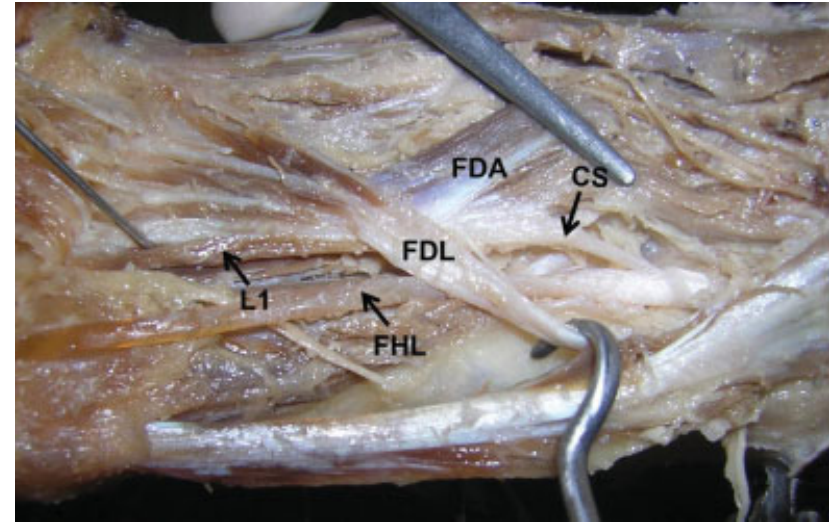

Fig. 2 Dissection of foot showing the cross link between the tendons of flexor hallucis longus and flexor digitorum longus. Abbreviations: FDL, flexor digitorum longus; FHL, flexor hallucis longus; FDA, flexor digitorum accessorius; L1, first lumbrical; CS, connecting slip.

great toe. In a study conducted on 64 limbs, it was found that the percentage of type I was $100 \%$; type II corresponded to $60.9 \%$; type III corresponded to $7.8 \%$; and type IV corresponded to $0 \%$. Out of the 64 cadavers, type la was found in 20 (31.3\%); type Ib was present in 39 cadavers (60.9\%); and type Ic was present in $5(7.8 \%){ }^{2}$ Similar results were also reported in other studies. ${ }^{6,7}$ According to the above description, the present case falls under the type Ia category; however, it is novel that the accessory slip received insertion of the FDA muscle and gave origin to the first lumbrical muscle.

The FDA shows variations in its origin and insertion. Sometimes, it may be reduced in size or it may be completely absent. Various insertion patterns of the FDA have been described in the literature. The FDA may be inserted to the lateral side of the flexor tendons or their undersurface. Its attachment may restrict only to the second and third toes; it may partly attach to the connecting slip of the FHL and to the tendons of the second, third and fourth toe; it may solely attach to the connecting slip of the FDL and FHL. ${ }^{8}$ In the present case, the FDA is mainly inserted into the connecting slip from the FHL and partly to the undersurface of the flexor tendons of the third and fourth toes. Macalister has reported the unusual origin of first lumbrical muscle from the tibial side of the FDL tendon to the second toe after receiving the connecting slip from the FHL. Rarely, it takes origin from the tibial side of the connecting slip of the FHL. ${ }^{8}$ In the present case, the first lumbrical had two heads that originated from both the FDL tendon and the FHL connecting slip.

Posterior tibial tendon deficiency (PTTD) is frequently treated with FDL tendon graft transfer along with associated bony procedures. A few authors have previously reported successful results with this procedure. ${ }^{9-11}$ However, there is a disagreement about the necessity of tenodesis of the leftover FDL to the FHL. A few authors have reported that FDL preservation is clinically irrelevant. Others have opined that the proximal portion of the FDL could be used for the tendon transfer as the flexion force of the toes could still be maintained by the FDA attachment to the distal FDL. ${ }^{2,12}$ In the present case, the FDA is attached to the connecting slip of the 
FHL and, therefore, in such cases, tenodesis of the FDL tendon to the FHL may be necessary to retain the function of the toes in PTTD treatment.

The FHL tendon is frequently used in the treatment of chronic Achilles tendinopathy. ${ }^{3}$ It has been suggested that after FHL tendon grafting, the distal portion of the FHL should be sutured to the FDL to preserve the flexion function of the hallux. ${ }^{2}$ As complex interconnections present between FHL and FDL, surgical transfer of any of these tendons would be a challenge, more so the one with anatomical variations. Unusual attachment of the FDA to the connecting slip of the FHL may interfere in the normal biomechanics of the FDL and FHL. Complex morphological variations involving the FHL, FDL, FDA and first lumbrical reported here may be useful for tendon reconstruction surgeons while planning surgical repair of Achilles tendon rupture and PTTD.

\section{References}

1 Standring S, Borley NR, Collins P, et al. Gray's Anatomy. Pectoral region, shoulder region and axilla. 40th ed. London: Churchill Livingstone; 2008, p. 2647-48

2 Mao H, Shi Z, Wapner KL, Dong W, Yin W, Xu D. Anatomical study for flexor hallucis longus tendon transfer in treatment of Achilles tendinopathy. Surg Radiol Anat 2015;37(06):639-647

3 Hahn F, Meyer P, Maiwald C, Zanetti M, Vienne P. Treatment of chronic achilles tendinopathy and ruptures with flexor hallucis tendon transfer: clinical outcome and MRI findings. Foot Ankle Int 2008;29(08):794-802
4 Schon LC, Shores JL, Faro FD, Vora AM, Camire LM, Guyton GP. Flexor hallucis longus tendon transfer in treatment of Achilles tendinosis. J Bone Joint Surg Am 2013;95(01):54-60

5 LaRue BG, Anctil EP. Distal anatomical relationship of the flexor hallucis longus and flexor digitorum longus tendons. Foot Ankle Int 2006;27(07):528-532

6 Plaass C, Abuharbid G, Waizy H, Ochs M, Stukenborg-Colsman C, Schmiedl A. Anatomical variations of the flexor hallucis longus and flexor digitorum longus in the chiasma plantare. Foot Ankle Int 2013;34(11):1580-1587

7 Hur MS, Kim JH, Woo JS, Choi BY, Kim HJ, Lee KS. An anatomic study of the quadratus plantae in relation to tendinous slips of the flexor hallucis longus for gait analysis. Clin Anat 2011;24(06): 768-773

8 Macalister A. On muscular anomalies in human anatomy, and their bearing upon homotypical myology. Proceedings of the Royal Irish Academy 1866-6910:126-164

9 Fayazi AH, Nguyen HV, Juliano PJ. Intermediate term follow-up of calcaneal osteotomy and flexor digitorum longus transfer for treatment of posterior tibial tendon dysfunction. Foot Ankle Int 2002;23(12):1107-1111

10 Myerson MS, Badekas A, Schon LC. Treatment of stage II posterior tibial tendon deficiency with flexor digitorum longus tendon transfer and calcaneal osteotomy. Foot Ankle Int 2004;25(07): 445-450

11 Schuh R, Gruber F, Wanivenhaus A, Hartig N, Windhager R, Trnka $\mathrm{HJ}$. Flexor digitorum longus transfer and medial displacement calcaneal osteotomy for the treatment of stage II posterior tibial tendon dysfunction: kinematic and functional results of fifty one feet. Int Orthop 2013;37(09):1815-1820

12 Trnka HJ. Dysfunction of the tendon of tibialis posterior. J Bone Joint Surg Br 2004;86(07):939-946 